\title{
Orientational Behavior of Ellipsoidal Silica- Coated Hematite Nanoparticles Integrated within an Elastomeric Matrix and its Mechanical Reinforcement
}

\author{
Antoni Sánchez-Ferrer, Raffaele Mezzenga, Hervé Dietsch*
}

The mechanical and orientational properties of IOENs consisting of integrated ellipsoidal SCH spindle-type nanoparticles within an elastomeric matrix are reported. The influence of the $\mathrm{SCH}$ surface chemistry, leading either to dispersed nanoparticles or crosslinked nanoparticles within the surrounding elastomeric matrix, is studied by mechanical uniaxial deformation (stress-strain) and SAXS measurements under stress. Without surface modifications, the $\mathrm{SCH}$ nanoparticles act as defects, and the Young's modulus of the elastomeric matrix remains unmodified. Surface-modified SCH nanoparticles acting as crosslinkers increase Young's modulus by a factor 1.2. SAXS measurements demonstrate that the integrated ellipsoidal nanoparticles orient upon a deformation larger than 50\% independently of the specific integration strategy.

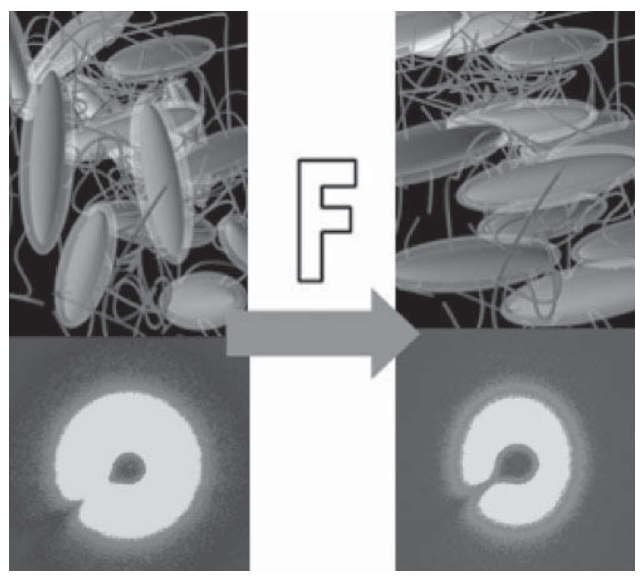

\section{Introduction}

Hybrid nanocomposite materials made out of integrated inorganic nanoparticles within an organic polymeric matrix can be found as gels, ${ }^{[1]}$ thermoplastics, ${ }^{[2]}$ thermosets or elastomers. ${ }^{[3]}$ This class of materials can exhibit the combined advantages of both the integrated nanoparticles, in terms of optical and electrical properties, and the processability of the polymeric matrix. As a result, bulk modification of the strength, stiffness, and toughness of the resulting materials are generally observed. ${ }^{[4,5]}$

A. Sánchez-Ferrer, R. Mezzenga

ETH Zurich, Institute of Food, Nutrition \& Health, Food \& Soft Materials Science, 8092 Zurich, Switzerland

H. Dietsch

Adolphe Merkle Institute and Fribourg Center for Nanomaterials, University of Fribourg, PO Box 209, 1723 Marly 1, Switzerland

E-mail: herve.dietsch@unifr.ch
Different approaches have been reported in the past for the integration of nanoparticles within a polymer network leading to nanocomposites, including particle/polymer hydrogels, ${ }^{[6]}$ particle/elastomer composites, ${ }^{[7-10]}$ silicathermoset networks via in situ polymerization, ${ }^{[11]}$ or linear polymer networks induced by particles. ${ }^{[12]}$ In most cases, aggregation and migration of the integrated fillers under an external trigger (e.g., electrical field, magnetic field, or a high shear rate) appears as a drawback of this specific route.

A potential solution is to integrate the nanoparticles in a covalent manner, binding them to the polymer backbone, which leads to a cooperative motion of both the continuous and dispersed phases. ${ }^{[3,11,13-14]}$ In this way, potential phase separation of the integrated nanoparticles can be avoided or reduced. Few examples can be found in the literature of integrating anisometric nanoparticles within a polymer matrix, and most of those are polymer/clay nanocomposites $^{[15-17]}$ or block copolymer/carbon nanotube nanocomposites, ${ }^{[18,19]}$ with improvement of mechanical properties, 
but still with aggregation of the nanoparticles in clusters. Two recent successful works on the integration of wires into a liquid-crystalline polymer network, ${ }^{[20]}$ and ellipsoidal nanoparticles into block copolymers ${ }^{[21]}$ were also reported. So far, no examples are available where anisometric nanoparticles are present in a chemical elastomeric network as crosslinkers, and the mechanical and orientational behavior of both nanoparticles and polymer matrix are studied in detail. In this context, a strategy towards inorganic/organic elastomer nanocomposites (IOENs) was also recently proposed using silica-coated hematite $(\mathrm{SCH})$ which was integrated within an elastomeric matrix. ${ }^{[22]}$ Evidence concerning the quality of the dispersion and distribution of the nanofillers within the matrix was obtained for surface-treated $\mathrm{SCH}$ with one of both functionalities taking part in the polyaddition process. ${ }^{[3]}$ However, the impact on the structural and mechanical properties provided by the presence of integrated nanoparticles within the elastomeric matrix was not disclosed, which is the main motivation for the present work.

In the current study we investigate whether integrated ellipsoidal nanoparticles can both mechanically reinforce an elastomeric matrix and orient under stress (i.e., coupling between the polymer network and the nanoparticles). To this end, nanoparticles acting as crosslinkers or mixed in an uncontrolled way have been compared at an equivalent filler concentration and evaluated by both classical mechanical tests and scattering patterns under mechanical stress.

\section{Experimental Part}

\section{Materials}

Iron(III) perchlorate hexahydrate $\left[\mathrm{Fe}\left(\mathrm{ClO}_{4}\right)_{3} \cdot 6 \mathrm{H}_{2} \mathrm{O}\right]$, poly(vinylpyrrolidone) (PVP, $10000 \mathrm{~g} \cdot \mathrm{mol}^{-1}$ ), and a $25 \%$ solution of tetramethylammonium hydroxide (TMAH) in methanol were all provided by Sigma-Aldrich and used as received. Sodium dihydrogen phosphate monohydrate $\left(\mathrm{NaH}_{2} \mathrm{PO}_{4} \cdot \mathrm{H}_{2} \mathrm{O}\right)$, urea, and absolute ethanol were provided by Fluka. Tetraethylorthosilicate (TEOS) and acetone were provided by Merck, and 3-aminopropyltriethoxysilane (APTES) from $A B C R$, were also used without further purification. Ultrapure water $(18.2 \mathrm{M} \Omega \cdot \mathrm{cm})$ purified by a Millio system, was used throughout the experiments. The diamine-terminated poly(propylene oxide) polymer Jeffamine D-2000 with approximate number-average molecular mass $\left(\bar{M}_{\mathrm{n}}\right)$ of $2000 \mathrm{~g} \cdot \mathrm{mol}^{-1}$ was kindly provided by Huntsman Corporation and degassed before use. The triisocyanate crosslinker Basonat HI- 100 was kindly provided by BASF SE and used as received.

\section{Synthesis of Ellipsoidal SCH Nanoparticles}

An aqueous solution of iron(III) perchlorate $(0.100 \mathrm{M})$, sodium phosphate monobasic $\left(5.5 \times 10^{-3} \mathrm{M}\right)$, and urea $(0.100 \mathrm{M})$ was kept in an oven at $98^{\circ} \mathrm{C}$ for $24 \mathrm{~h}$. The precipitated nanoparticles were then washed five times by centrifugation at $10000 \mathrm{rpm}$ for $15 \mathrm{~min}$ followed by redispersion in water using an ultrasonic bath. Nanoparticles $(150 \mathrm{mg})$ were dispersed in a solution of PVP (surface density of 13 PVP molecules $\cdot \mathrm{nm}^{-2}$ ). The suspension was stirred for $12 \mathrm{~h}$ to let the polymer adsorb onto the surface. The stabilized nanoparticles were then transferred in $485 \mathrm{~mL}$ of a 17.497:82.488:0.015 v/v mixture of water/ethanol/tetramethylammonium chloride ( $25 \%$ solution in methanol). While stirring and sonicating, a 1:2 mixture of TEOS and ethanol was added in three portions of $6 \mathrm{~mL}$ each every $20 \mathrm{~min} \cdot{ }^{[23,24]}$ For surface-functionalized nanoparticles, $30 \mathrm{~min}$ after the last TEOS addition, $160 \mu \mathrm{L}$ of APTES was added. Sonication was applied for $2 \mathrm{~h}$ after the last injection and the mechanical stirring was further continued overnight at room temperature. The nanoparticles were then washed by centrifugation and redispersion. After adding acetone to the aqueous nanoparticle dispersion, the precipitate was washed five times by centrifugation at $10000 \mathrm{rpm}$ for $15 \mathrm{~min}$, followed by redispersion in acetone using an ultrasonic bath. The final concentrations of functionalized SCH nanoparticles and normal $\mathrm{SCH}$ nanoparticles were 1.4 and $2.8 \mathrm{mg} \cdot \mathrm{mL}^{-1}$, respectively. The synthetic route to obtain the ellipsoidal SCH nanoparticles, i.e., the crosslinkable functionalized nanoparticles (FNPs) and non-crosslinkable nanoparticles (NPs), is shown in Scheme 1.

From scattering and transmission electron microscopy (TEM) experiments, ${ }^{[3,25]}$ the prolate spheroid nanoparticle $(a>b=c)$ radii were calculated, and for the FNPs, values of $a=129 \mathrm{~nm}$ and $b=26 \mathrm{~nm}$ were found. The NPs had radii of $a=114 \mathrm{~nm}$ and $b=23 \mathrm{~nm}$. Both types of nanoparticles had a shell thickness of $\mathrm{s}=4.8 \mathrm{~nm}$. The polydispersity of the hematite core was 0.5 , while that for the silica shell was 0.2 .

\section{Synthesis of IOENs}

Three elastomers were synthesized: a reference elastomer (E0) without any nanoparticle, and two elastomers containing SCH nanoparticles (E1 and E2). The two SCH nanoparticles in the matrix were either functionalized with amino groups on the surface of the silica-coated hematite (FNP for E1), or bare silica coated hematite nanoparticles (NP for E2). For the synthesis of the three elastomers, two solutions in acetone were prepared: one containing the diamino-terminated polymer, and the other the trifunctionalized crosslinker with or without nanoparticles. In order to obtain a final solid content of $15 \% \mathrm{w} / \mathrm{v}, 3.85 \mathrm{~g}$ of Jeffamine D-2000 was dissolved in $11.1 \mathrm{~mL}$ of acetone, and $0.65 \mathrm{~g}$ of Basonat $\mathrm{HI}-100$ was dissolved in $14.5 \mathrm{~mL}$ of acetone. In the case of elastomers containing $\mathrm{SCH}$ nanoparticles, the Basonat $\mathrm{HI}-100$ was dissolved in an acetone dispersion of the corresponding nanoparticles to be incorporated. The two solutions were mixed and gently stirred for $5 \mathrm{~min}$, and

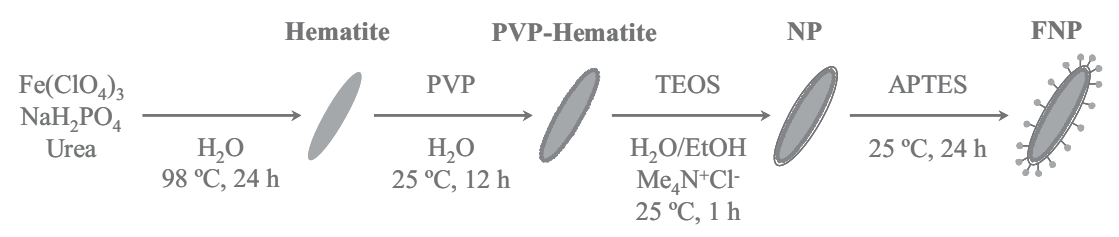

Scheme 1. Synthetic route to obtain the ellipsoidal SCH nanoparticles (NP: non-crosslinkable nanoparticles; FNP: crosslinkable functionalized nanoparticles). 

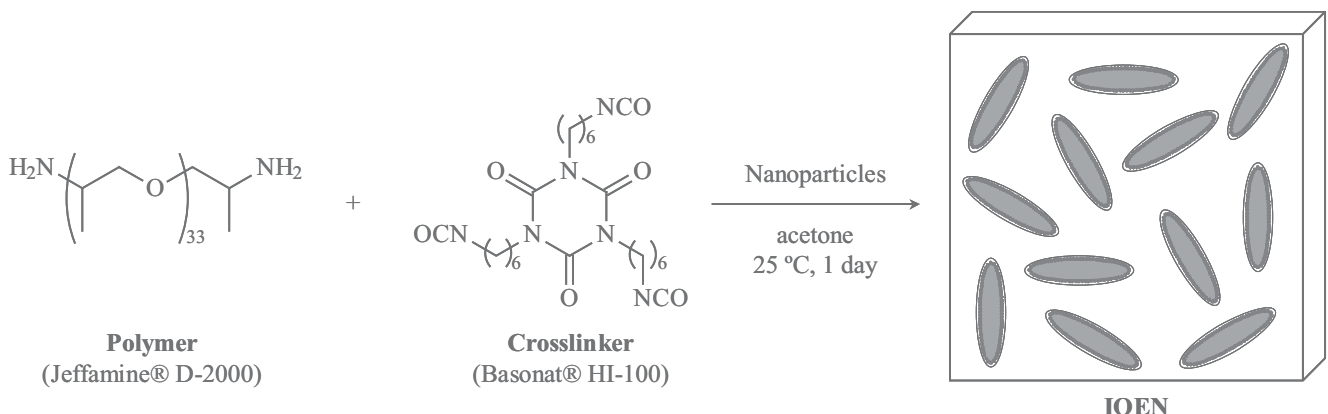

Scheme 2. Chemical structures of the organic components for the synthesis of IOENs.

the final solution was cast onto the glass surface of a Petri dish. One day after the samples were cast, the obtained film was allowed to dry in the atmosphere and peeled from the surface. ${ }^{[3,22]}$ Samples were cut from their corresponding free-standing films $\left(14.6 \times 5.0 \times 0.75 \mathrm{~mm}^{3}\right)$.

The theoretical concentrations of nanoparticles in the two samples were $c(\mathrm{E} 1)=0.48 \mathrm{wt} . \%$ and $c(\mathrm{E} 2)=0.88 \mathrm{wt} . \%$, which correspond to 0.11 and 0.20 vol.-\%, respectively. These concentrations correspond to $2.1 \times 10^{12}$ and $5.5 \times 10^{12}$ particles $\cdot \mathrm{cm}^{-3}$, respectively. For a homogeneous distribution of nanoparticles, these concentrations lead to a distance between two neighboring nanoparticles in the elastomeric matrix of about 500-700 and 300$500 \mathrm{~nm}$, respectively (the lower limit corresponds to the long axis distance and the upper limit to the short axis distance). The materials and preparation of the inorganic nanoparticles, as well as their integration into the polymer network, is depicted in Scheme 2. Figure 1 presents the films and their optical and TEM pictures, where sample E1 shows a good dispersion of the FNPs.

\section{Apparatus and Techniques}

Uniaxial stress-strain measurements were performed with a Linkam TST350 thermostatted tensile testing system and controlled by a T95-LinkSys controller. Samples were stretched by two

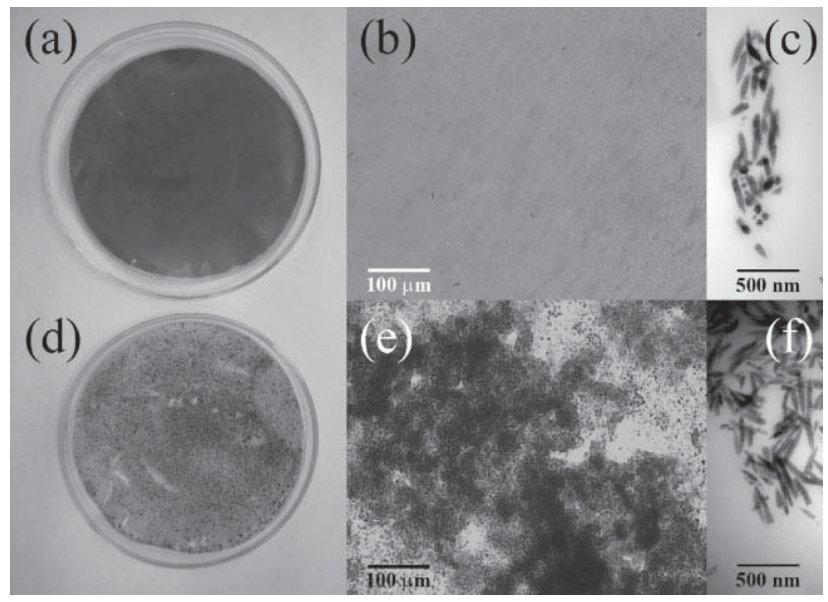

Figure 1. a) Sample E1 and its corresponding optical microscopy image (b) and TEM image (c). d) Sample E2 and its corresponding optical microscopy image (e) and TEM image (f). controlled microstep motors at an extension rate of $10 \mu \mathrm{m} \cdot \mathrm{min}^{-1}$, which corresponded to a strain rate of $1.14 \times 10^{-5} \mathrm{~s}^{-1}$. The stress was measured by a transducer load cell (tensile force of $20 \mathrm{~N}$; force resolution $0.001 \mathrm{~N})$. All relevant data such as temperature, uniaxial strain ratio $\left(\lambda=L / L_{0}\right.$, where $L$ and $L_{0}$ are the lengths of the film in the stretched and nonstretched states), and nominal uniaxial stress $\left(\sigma_{\mathrm{n}}\right)$ were continuously logged by Linksys $32 \mathrm{AV}$ software.

Simultaneous small and wide-angle X-ray scattering (SAXS and WAXS, respectively) experiments were performed using a Rigaku MicroMax-002+ microfocused beam ( $4 \mathrm{~kW}, 45 \mathrm{kV}, 0.88 \mathrm{~mA})$ in order to obtain direct information on the SAXS and WAXS reflections. The

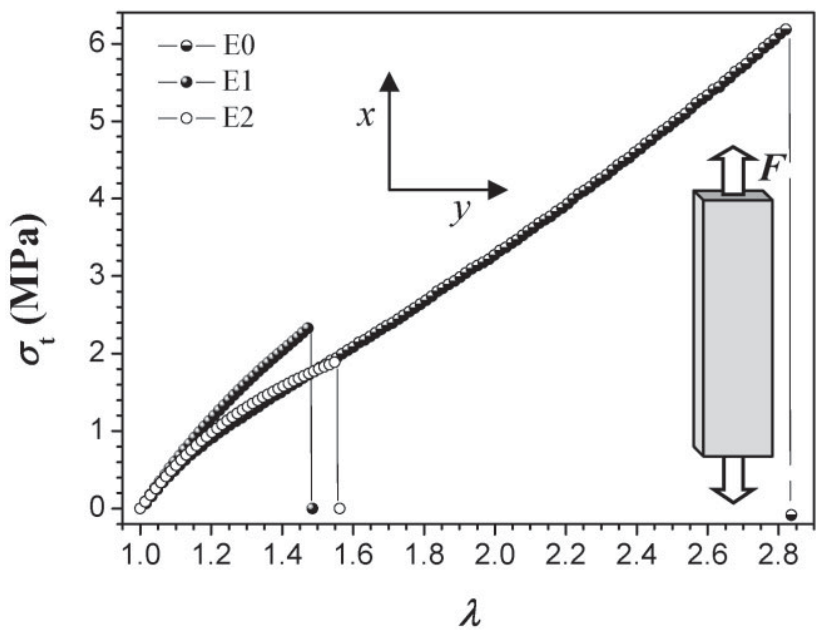

Figure 2. Uniaxial stress-strain curves for the three elastomeric nanocomposites EO, E1, and E2, and definition of the direction of deformation. Note: $\sigma_{\mathrm{t}}=\lambda \cdot \sigma_{\mathrm{n}}$

Table 1. Young's modulus $(E)$, maximum strain $\left(\lambda_{\max }\right)$, energy at $\lambda=1.48(U)$, and toughness $\left(U_{\max }\right)$ for the three elastomeric samples.

\begin{tabular}{|c|c|c|c|c|}
\hline \multirow[t]{2}{*}{ Sample } & $\boldsymbol{E}$ & $\lambda_{\max }$ & $U(\lambda=1.48)$ & $U_{\max }$ \\
\hline & $\mathrm{MPa}$ & & $\mathrm{J} \cdot \mathrm{cm}^{-3}$ & $\mathrm{~J} \cdot \mathrm{cm}^{-3}$ \\
\hline E0 & $5.1 \pm 0.1$ & 2.83 & 0.47 & 5.68 \\
\hline E1 & $6.2 \pm 0.1$ & 1.48 & 0.62 & 0.62 \\
\hline E2 & $5.3 \pm 0.1$ & 1.56 & 0.50 & 0.64 \\
\hline
\end{tabular}


$\mathrm{Cu} \mathrm{K}_{\alpha}$ radiation $\left(\lambda_{\mathrm{Cu} \mathrm{K} \alpha}=1.5418 \AA\right)$ was collimated by three pinhole $(0.4,0.3$, and $0.8 \mathrm{~mm})$ collimators. The incident beam was normal to the surface of the film. The scattered X-ray intensity was detected by a Fuji Film BAS-MS 2025 imaging plate system $\left(15.2 \times 15.2 \mathrm{~cm}^{2}\right.$, $50 \mu \mathrm{m}$ resolution) and a two-dimensional Triton-200 X-ray detector $(20 \mathrm{~cm}$ diameter, $200 \mu \mathrm{m}$ resolution). An effective scatteringvector range of $0.05 \mathrm{~nm}^{-1}<q<25 \mathrm{~nm}^{-1}$ was obtained, where $q$ is the scattering wavevector defined as $q=4 \pi \sin \theta / \lambda_{\mathrm{Cu} K \alpha}$, with a scattering angle of $2 \theta$. From the scattering intensities, the order parameter $(S)$ was defined according to Lovell and Mitchell. ${ }^{[26,27]}$

A Leica DM LB optical microscope equipped with a Linkam CSS450 hot-stage was used to analyze the homogeneity of the samples.

TEM images were obtained with a Philips TEM (CM 100) instrument operated at $80 \mathrm{kV}$. The elastomeric samples were cryo-ultramicrotomed at $-80^{\circ} \mathrm{C}$ using a diamond knife on a Leica Ultracut UCT Ultramicrotome to give $50 \mathrm{~nm}$ thick sections. Sections were then transferred onto 600-mesh carbon-coated copper grids.

\section{Results and Discussion}

\section{Uniaxial Deformations}

Mechanical deformations are a very suitable way to understand the effect of nanoparticles as reinforcement in polymeric matrices. Furthermore, the combination of uniaxial stress-strain experiments together with X-ray measurements is a powerful technique to understand both the structural and mechanical processes taking place during deformation.

The resulting curves are presented in Figure 2, where the reference sample (E0) shows a common elastomeric behavior. In the elastic region, during the first deformation steps, the Young's modulus can be calculated following the relation $E=\mathrm{d} \sigma / \mathrm{d} \lambda$. In this way, the two samples containing nanoparticles (E1 and E2) and the reference sample were uniaxially deformed, and the restoring force measured at each stretching step. The evaluation of the initial slopes for each sample shows a difference when FNPs or NPs are present in the hybrid nanocomposite. The elastomer containing FNPs (E1) has a Young's modulus of $E_{1}=6.2 \pm 0.1 \mathrm{MPa}$,
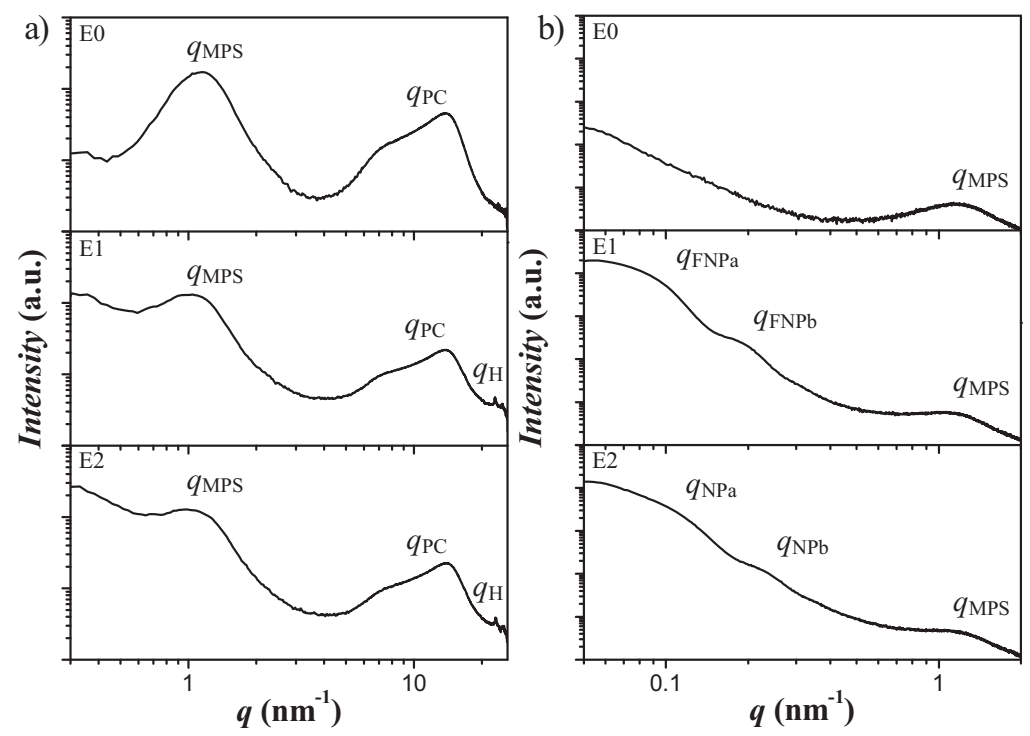

Figure 3. a) WAXS and b) SAXS radial scattering distributions for the three elastomeric samples EO, E1, and E2.

whereas the elastomer with NPs (E2) has a Young's modulus $E_{2}=5.3 \pm 0.1 \mathrm{MPa}$, similar to the corresponding value for the reference elastomer (EO) of $E_{0}=5.1 \pm 0.1 \mathrm{MPa}$. This difference of $20 \%$ in the Young's modulus for the elastomer E1 with respect to the elastomer E2 shows how the nanoparticles as crosslinkers enhance the mechanical properties of the nanocomposite.

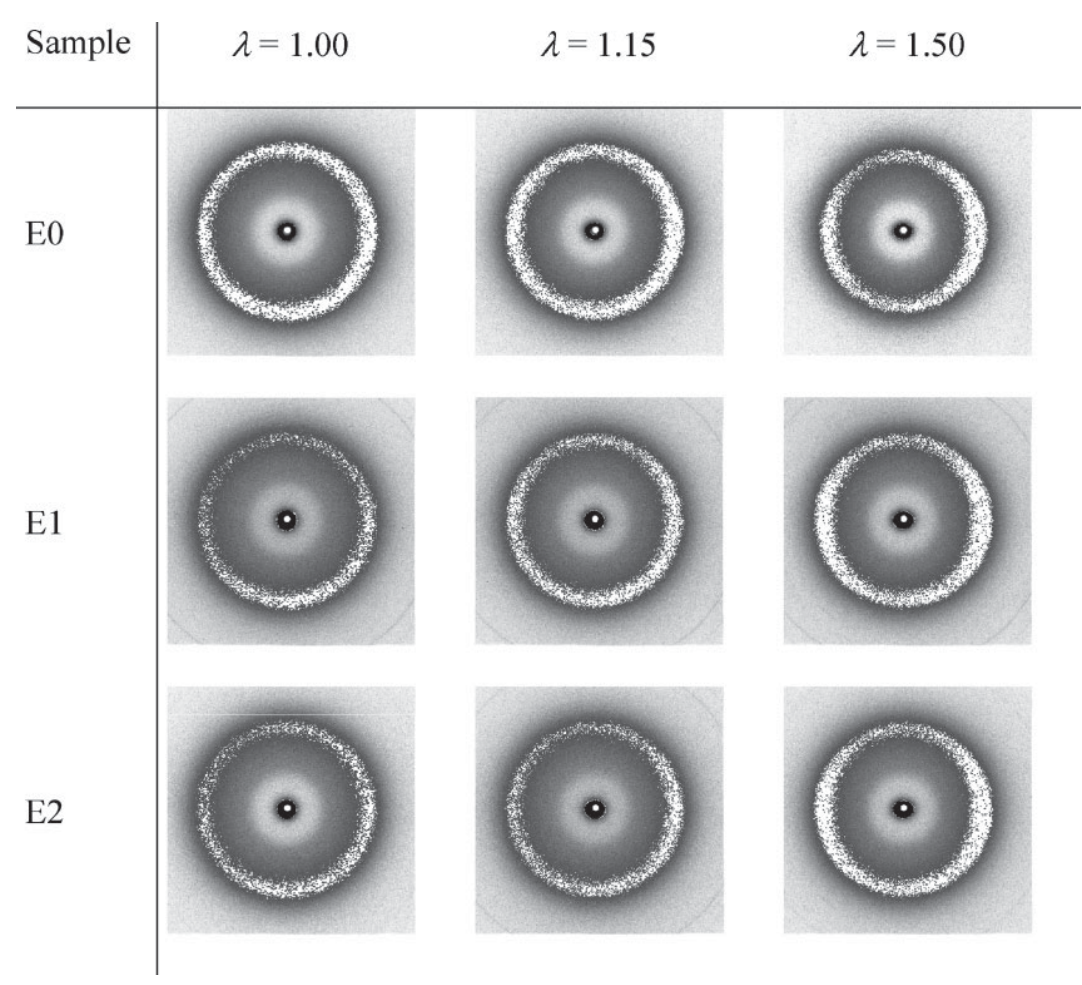

Figure 4. 2D WAXS patterns for the three elastomeric samples Eo, E1, and E2 at the strain values of $\lambda=1.0,1.15$, and 1.50 . 
Furthermore, the presence of both kinds of nanoparticle makes the elastomer more brittle as can be observed by the maximum elongation during deformation. The pure reference system (EO) could be drawn up to strain values of $\lambda_{\max }=2.83$, while for the nanocomposites only values of $\lambda_{\max }=1.48$ for $\mathrm{E} 1$ and $\lambda_{\max }=1.56$ for E2 were obtained. The toughness, i.e., energy applied until rupture of the sample, of the reference elastomer $\left(5.7 \mathrm{~J} \cdot \mathrm{cm}^{-3}\right)$ differs considerably (800\%) with respect to the values for both IOENs $\left(0.6 \mathrm{~J} \cdot \mathrm{cm}^{-3}\right)$. This indicates that the nanoparticles act as defects for crack initiation, reducing the toughness of the elastomeric matrix.

In order to evaluate and compare the efficiency and energy needed during the deformation process for the three samples, the area under each stress/strain curve at fixed strain was calculated. The energy at $\lambda=1.48$, the maximum elongation for sample E1, shows noticeable differences between the samples. The energy for the sample with FNPs (E1) is $32 \%$ higher than for the reference sample (E0), whereas for the sample with NPs (E2) it is only 6\%, providing further evidence for mechanical enhancement of the crosslinkable nanoparticles in the nanocomposite. In Table 1 , the Young's modulus $(E)$, maximum strain $\left(\lambda_{\max }\right)$, energy at $\lambda=1.48(U)$, and toughness $\left(U_{\max }\right)$ for the three elastomeric samples are summarized.

\section{Mechanical Coupling between Polymers and Nanoparticles}

The next point assessed is whether any coupling between the polymer matrix and the nanoparticles exists, that is, if the external mechanical field which orients the polymer backbones induces any kind of alignment to the nanoparticles. In order to observe any potential effect, both nanocomposites (E1 and E2) as well as the reference elastomer EO were analyzed by SAXS (in the region where the nanoparticles diffuse, $\left.0.055 \mathrm{~nm}^{-1}<q_{\mathrm{FNP}}-q_{\mathrm{NP}}<0.15 \mathrm{~nm}^{-1}\right)$ and by WAXS experiments (where the polymer chains interact and align, $6.0 \mathrm{~nm}^{-1}<$ $q_{\mathrm{PC}}<20 \mathrm{~nm}^{-1}$ ) during the stretching process.

In the WAXS region (Figure 3a), the elastomeric matrix already shows

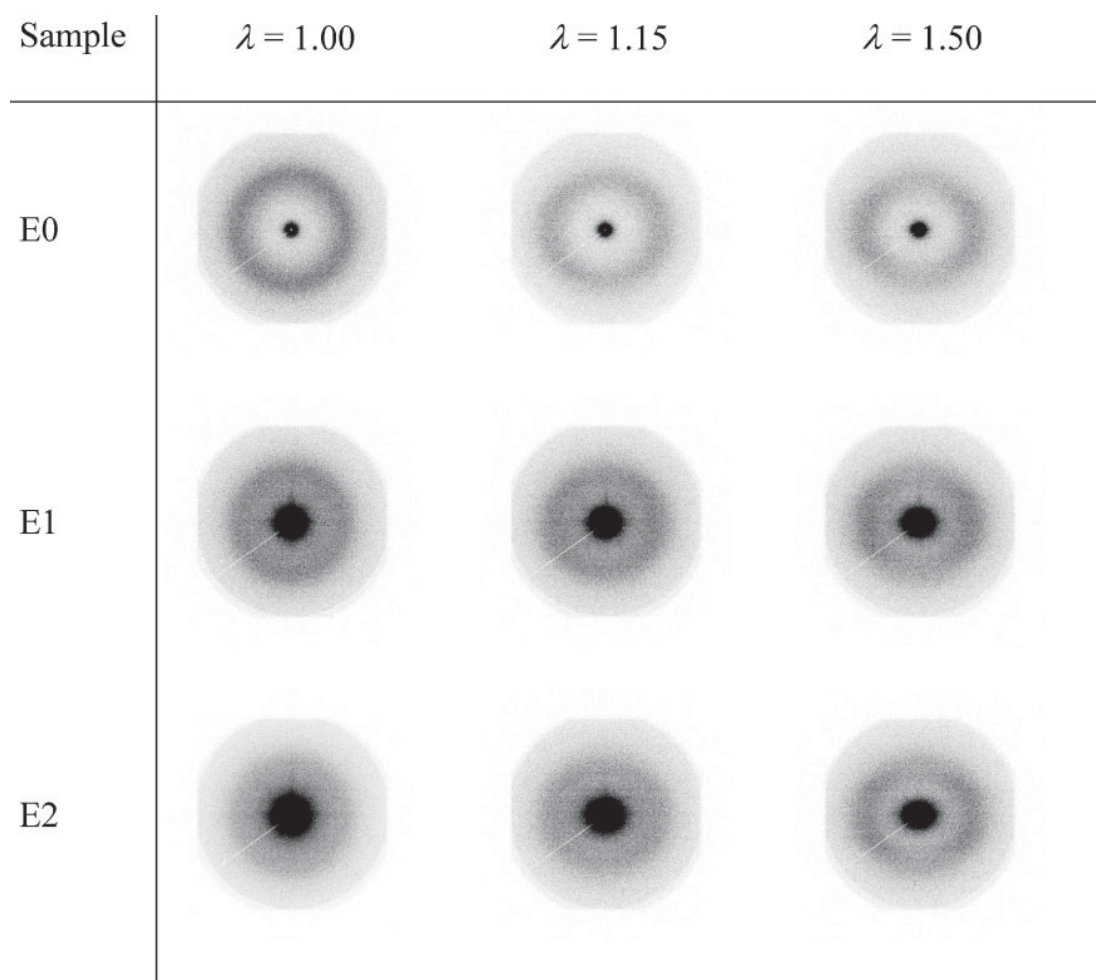

Figure 5. 2D SAXS patterns for the three elastomeric samples EO, E1, and E2 at the strain values of $\lambda=1.0,1.15$ and 1.50 .

scattering peaks at $q_{\mathrm{PC}}=14.4 \mathrm{~nm}^{-1}\left(d_{\mathrm{PC}}=0.44 \mathrm{~nm}\right)$ and $q_{\mathrm{MPS}}=1.20 \mathrm{~nm}^{-1}\left(d_{\mathrm{MPS}}=5.2 \mathrm{~nm}\right)$. The first peak $q_{\mathrm{PC}}$ is related to the polymer backbone chains distance. The second peak $q_{\text {MPS }}$ is the disordered microphase separated domains between the aliphatic segments of the crosslinker and the ether segments of the polymer chains. This second scattering peak is enhanced by the presence of hydrogen

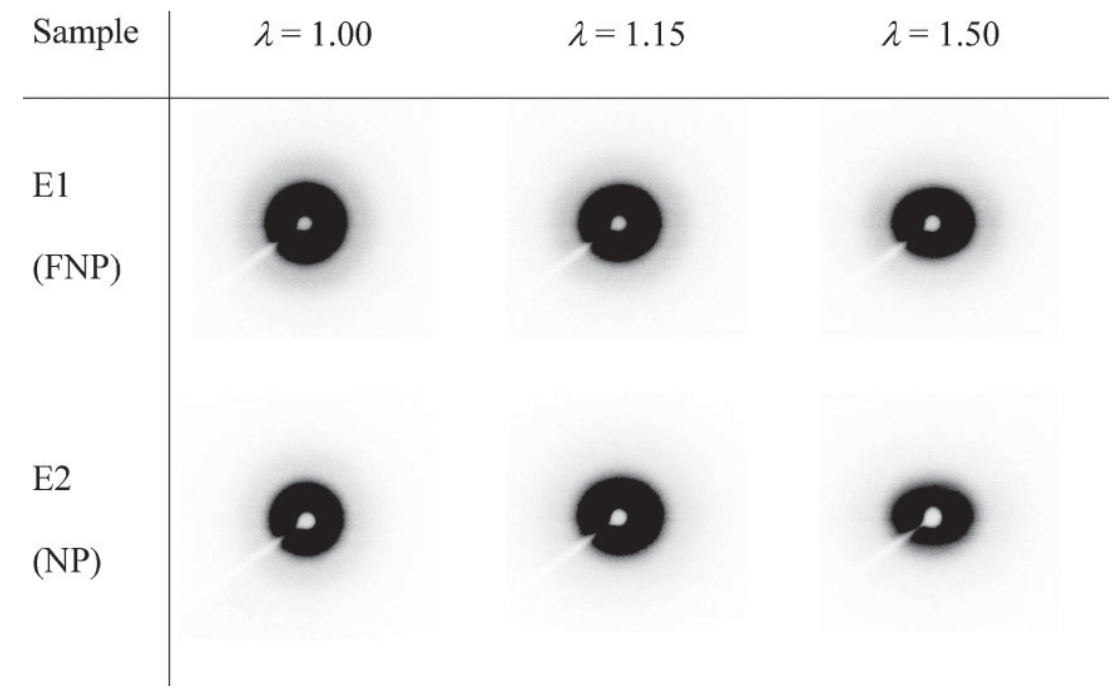

Figure 6. 2D SAXS patterns of the nanoparticles (FNP and NP) at the strain values of $\lambda=1.0,1.15$ and 1.50 . 
bonding of the urea motifs created during the condensation polymerization process. ${ }^{[22]}$ The two IOEN samples also show scattering peaks at $q_{\mathrm{H}}=16.9,23.1$, and $24.8 \mathrm{~nm}^{-1}$, which correspond to the $\alpha-\mathrm{Fe}_{2} \mathrm{O}_{3}$ nanoparticles in their hematite form.

In the SAXS region (Figure $3 b$ ), the two IOEN samples show two scattering peaks related to the anisotropic shape of the ellipsoidal SCH nanoparticles. The peaks $q_{\mathrm{FNPa}}$ and $q_{\mathrm{NPa}}$ correspond to the long $a$-axis of the nanoparticles, and the peaks $q_{\mathrm{FNPb}}$ and $q_{\mathrm{NPb}}$ to the $b$-axis. The FNPs have lower peak values, i.e., greater distances, for both axes with respect to the NPs. This difference is attributable to the layer thickness which contains the PVP and silica layer, plus the functional groups that reacted with the polymer matrix.

Experiments were carried out at strain values of $\lambda=1.00,1.15$, and 1.50 (Figure 4 and 5). Already the 2D scattering patterns show insightful information for both samples: i) the samples are becoming aligned upon stretching in the same direction parallel to the applied stress and ii) the intensity of the crosslinkable nanoparticles is much higher than the non-crosslinkable ones (Figure 6). This can be easily explained by the better dispersion achievable with FNPs with respect to NPs, which exhibit a higher tendency to aggregate, as shown in our previous work. ${ }^{[3]}$

The azimuthal Gaussian distribution of all scattering peaks ( $q_{\mathrm{PC}}, q_{\mathrm{MPS}}, q_{\mathrm{FNPb}}$, and $q_{\mathrm{FNPa}}$ ) allows evaluation of the order in the sample in terms of the polymer matrix and the nanoparticles (Figure 7). The orientational order parameter $S$ is calculated based on the average of the second Legendre polynomial according to the literature ${ }^{[26,27]}$ from the azimuthal scattering intensity distribution $I(\varphi)$ as:

$$
\begin{aligned}
S & =-2 P_{2}=-2\left\langle\frac{3 \cos ^{2} \varphi-1}{2}\right\rangle \\
& =-\frac{\int_{0}^{\pi / 2} I(\varphi)\left(3 \cos ^{2} \varphi-1\right) \sin \varphi \mathrm{d} \varphi}{\int_{0}^{\pi / 2} I(\varphi) \sin \varphi \mathrm{d} \varphi}
\end{aligned}
$$
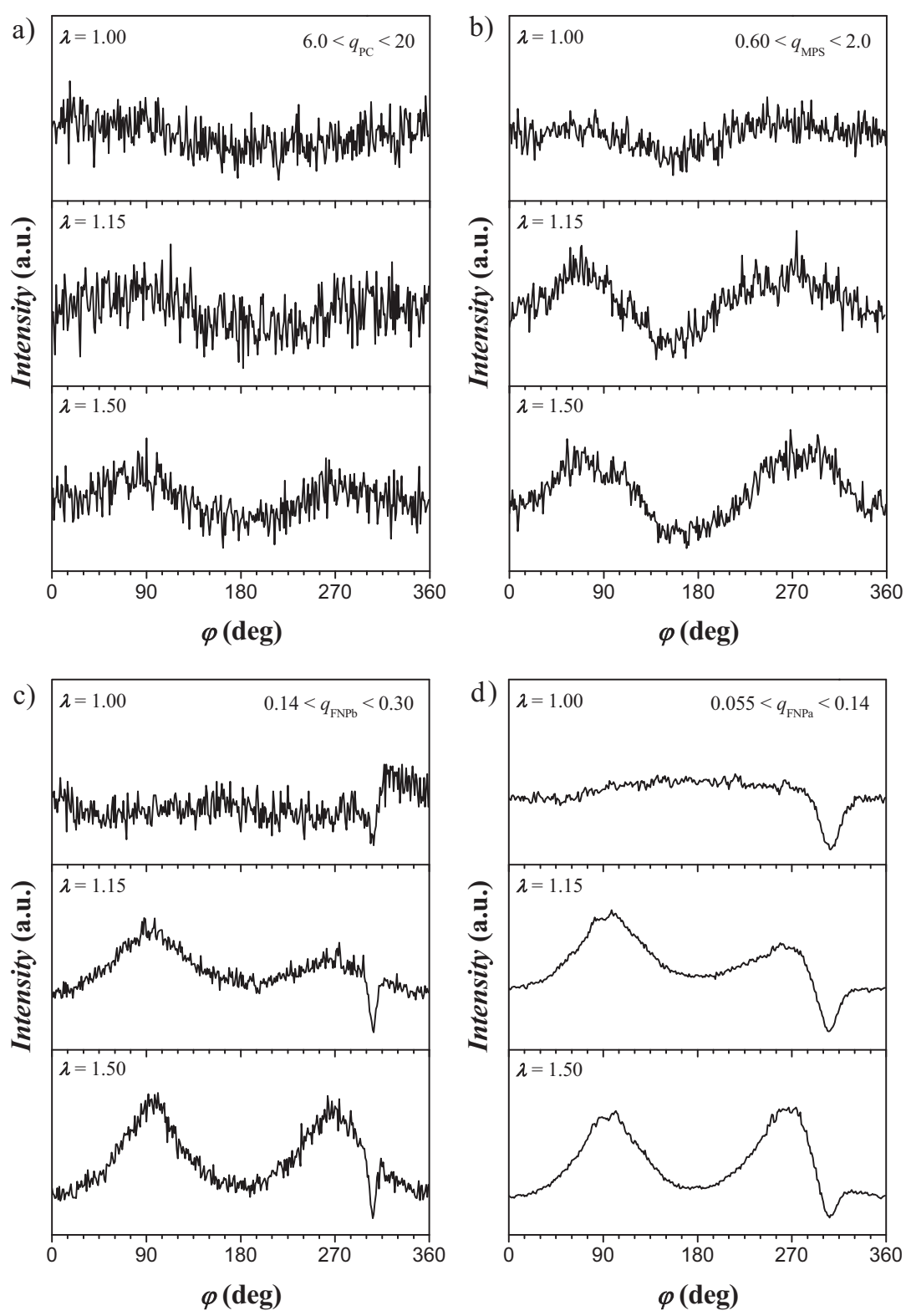

Figure 7. Azimuthal scattering distributions of the sample $\mathrm{E}_{1}$ at a) $6.0 \mathrm{~nm}^{-1}<q_{\mathrm{PC}}<$ $20 \mathrm{~nm}^{-1}$, b) $0.60 \mathrm{~nm}^{-1}<q_{\mathrm{MPS}}<2.0$, c) $0.14 \mathrm{~nm}^{-1}<q_{\mathrm{FNPb}}<0.30 \mathrm{~nm}^{-1}$, and d) $0.055 \mathrm{~nm}^{-1}<$ $q_{\mathrm{FNPa}}<0.14 \mathrm{~nm}^{-1}$ at the strain values of $\lambda=1.00,1.15$ and 1.50 .

In Figure 8, the evolution of the orientational order parameters as a function of the applied strain is presented for the three elastomeric samples at the different scattering regions. The orientational order parameter of the polymer chains (Figure $8 \mathrm{a}$ ) goes up to $S=0.33,0.24$, and 0.31 , and the orientational order parameter of the micro-phase separated domains (Figure $8 b$ ) up to $S=0.35,0.37$, and 0.35 for the samples E0, E1, and E2, respectively, when the strain reaches $\lambda=1.50$. The orientational order parameter for the nanoparticles (Figure $8 c$ and $8 d$ ) increases faster than the previous ones when stretching the samples, reaching values of $S=0.50-0.51$ and $0.56-0.53$ for the two IOEN 


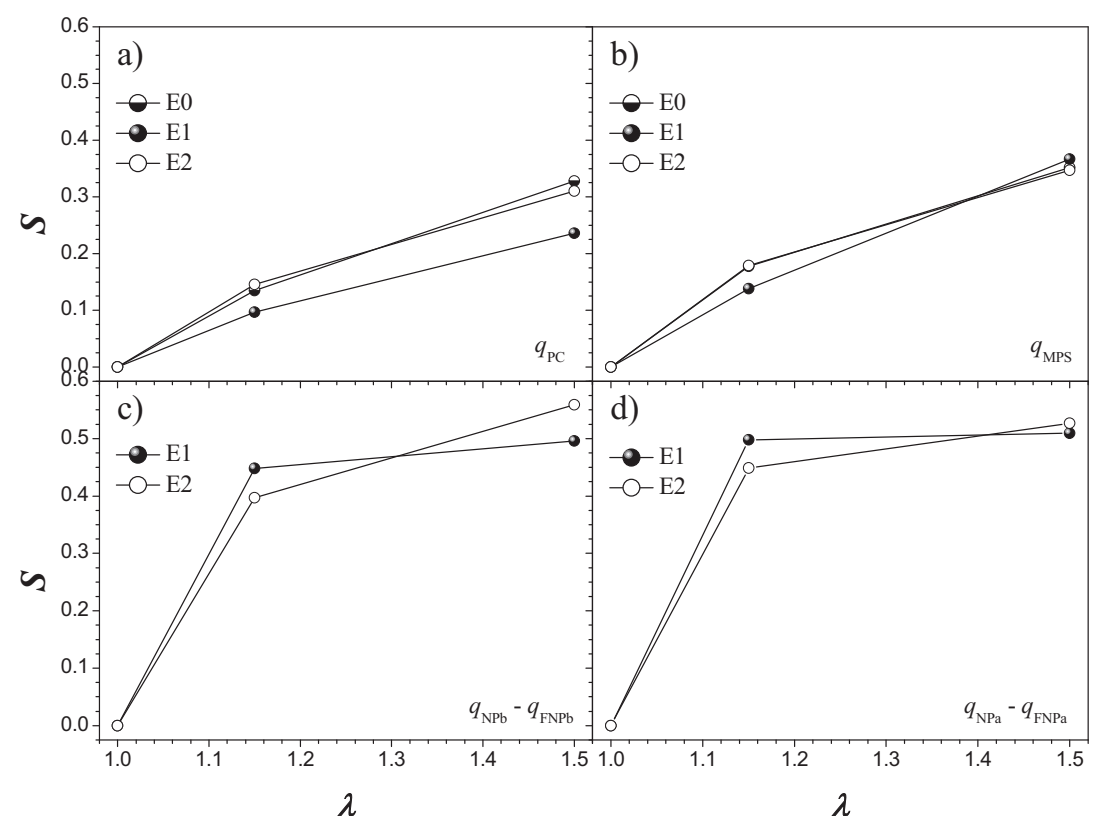

Figure 8. Order parameter $(S)$ as a function of the strain $(\lambda)$ for the three elastomeric samples at a) $6.0 \mathrm{~nm}^{-1}<q_{\mathrm{PC}}<20 \mathrm{~nm}^{-1}$, b) $0.60 \mathrm{~nm}^{-1}<q_{\mathrm{MPS}}<2.0$, c) $0.14 \mathrm{~nm}^{-1}<q_{\mathrm{FNPb}}<$ $0.30-0.16 \mathrm{~nm}^{-1}<q_{\mathrm{NPb}}<0.33 \mathrm{~nm}^{-1}$, and d) $0.055 \mathrm{~nm}^{-1}<q_{\mathrm{FNPa}}<0.14-0.055 \mathrm{~nm}^{-1}<q_{\mathrm{NPa}}<$ $0.16 \mathrm{~nm}^{-1}$. nanoparticle-induced fracture initiation: this resulted in a maximum achievable deformation of $50 \%(\lambda=1.50)$ prior to fracture.

Evidence of the strain-induced orientation of the ellipsoidal-shaped nanoparticles was provided by the SAXS pattern of the nanocomposite under mechanical stress. Independent of the type of interaction between the nanoparticles and the elastomeric matrix, coupling between the polymer network and the nanoparticles was confirmed, as shown by the values of their orientational order parameters.

Acknowledgements: The authors acknowledge the financial support provided by the Adolphe Merkle Foundation and thank the Swiss National Foundation NFP62 "Smart Materials" for funding the project. samples E1 and E2, respectively. It seems that less stretching is needed for the orientation of the nanoparticles compared with both the inter-chain and inter-domains distances.

By analyzing the azimuthal distribution of the scattering intensities at different strain values for both IOEN samples, it is apparent that they essentially align in an identical way irrespective of their chemical (E1) or physical (E2) adhesion to the polymer matrix. Thus, it can be inferred that large nanoparticles such as those used in the present study are fully coupled to the polymer network and any deformation in the elastomeric matrix induces and controls their alignment and the resulting mechanical properties.

\section{Conclusion}

We have demonstrated the physical interactions between nanoparticles and the polymer matrix in a hybrid nanocomposite formed by an elastomeric matrix loaded with ellipsoidal SCH nanoparticles. An increase of $20 \%$ for the Young's modulus was demonstrated from the integration of 0.5 wt.-\% ellipsoidal nanoparticles acting as crosslinking agents. At an equivalent concentration, surfaceuntreated nanoparticles were found to act as defects, and the Young's modulus of their corresponding elastomer was barely increased with respect to the reference sample. Both nanocomposites exhibited increased brittleness as compared to the reference matrix, as a consequence of
Keywords: elastomers; hematite nanoparticles; nanocomposites; stress/strain; X-ray scattering

[1] Y. J. Gong, M. Y. Gao, D. Y. Wang, H. Möhwald, Chem. Mater. 2005, 17, 2648.

[2] H. R. Dennis, D. L. Hunter, D. Chang, S. Kim, J. L. White, J. W. Cho, D. R. Paul, Polymer 2001, 42, 9513.

[3] A. Sánchez-Ferrer, M. Reufer, R. Mezzenga, P. Schurtenberger, H. Dietsch, Nanotechnology 2010, 21, 185603.

[4] B. M. Novak, Adv. Mater. 1993, 5, 422.

[5] M. Zrínyi, L. Barsi, A. Büki, J. Chem. Phys. 1996, 104, 8750.

[6] D. Collin, G. K. Auernhammer, O. Gavat, P. Martinoty, H. R. Brand, Macromol. Rapid Commun. 2003, 24, 737.

[7] C. Sena, M. H. Godinho, A. M. Figueiredo-Neto, J. Appl. Phys. 2007, 102, 073524.

[8] A. M. Figueiredo-Neto, M. H. Godinho, T. Toth-Katona, P. Palffy-Muhoray, Braz. J. Phys. 2005, 35, 184.

[9] A. Ghosh, N. K. Sheridon, P. Fischer, Small 2008, 4, 1956.

[10] A. Kaiser, M. Winkler, S. Krause, H. Finkelmann, A. M. Schmidt, J. Mater. Chem. 2009, 19, 538.

[11] M. Saric, H. Dietsch, P. Schurtenberger, Colloid Surf. A 2006, 291, 110.

[12] A. Serbescu, K. Saalwächter, Polymer 2009, 50, 5434.

[13] R. Fuhrer, E. K. Athanassiou, N. A. Luechinger, W. J. Stark, Small 2009, 5, 383.

[14] H. Dietsch, V. Malik, M. Reufer, C. Dagallier, A. Shalkevich, M. Saric, T. Gibaud, F. Cardinaux, F. Scheffold, A. Stradner, P. Schurtenberger, Chimia 2008, 62, 805.

[15] A. Okada, A. Usuki, Macromol. Mater. Eng. 2006, 291, 1449. 
[16] S. Pradhan, F. R. Costa, U. Wagenknecht, D. Jehnichen, A. K. Bhowmick, G. Heinrich, Eur. Polym. J. 2008, 44, 3122.

[17] H. S. Lee, P. D. Fasulo, W. R. Rodgers, D. R. Paul, Polymer 2005 , 46, 11673.

[18] A. Kelarakis, K. Yoon, I. Sics, R. H. Somani, B. S. Hsiao, B. Chu, Polymer 2005, 46, 5103.

[19] Y. Li, H. Shimizu, Macromolecules 2009, 42, 2587.

[20] Y. Y. Huang, J. Biggins, Y. Ji, E. M. Tenrentjev, J. Appl. Phys. 2010, 107, 083515.

[21] M. R. Hammond, H. Dietsch, O. Pravaz, P. Schurtenberger, Macromolecules 2010, 43, 8340.
[22] A. Sánchez-Ferrer, D. Rogez, P. Martinoty, Macromol. Chem. Phys. 2010, 211, 1712.

[23] M. Ocaña, M. P. Morales, C. J. Serna, J. Colloid Interface Sci. 1999, 212, 317.

[24] S. Sacanna, L. Rossi, B. Kuipers, A. Philipse, Langmuir 2006, 22, 1822.

[25] M. Reufer, H. Dietsch, U. Gasser, A. M. Hirt, A. Menzel, P. Schurtenberger, J. Phys. Chem. B 2010, 114, 4763.

[26] R. Lovell, G. R. Mitchell, Acta Crystallogr. A 1981, 37, 135.

[27] G. R. Mitchell, A. H. Windle, "Development in Crystalline Polymers-2", D. C. Basset, Ed., Elsevier Applied Science, London 1988, Vol. 3, p. 115. 